\title{
Gobernanza económica global: ¿Dónde estamos?
}

\section{FEDERICO STEINBERG}

Universidad Autónoma de Madrid, Facultad de CC.EE., c/ Francisco Tomas y Valiente, 5, Campus de Cantoblanco, 28049 Madrid, España. E-mail: federico.steinberg@uam.es

\section{RESUMEN}

Este artículo analiza los retos a los que se enfrenta la comunidad internacional para construir una gobernanza económica global, efectiva y legítima. Tras analizar la justificación normativa para la coordinación económica internacional y los obstáculos para alcanzarla, se procede a explorar los avances y asignaturas pendientes en las distintas áreas económicas relevantes. Para ello, se analiza el papel del G-20, que desde 2008 ha emergido como en el epicentro de la coordinación económica internacional.

Palabras clave: Gobernanza económica, cooperación internacional, proteccionismo, guerra de divisas, FMI.

\section{Global Economic Governance: Where do we Stand?}

\begin{abstract}
This paper analyzes the challenges that the international community faces to build an effective and legitimate global economic governance. After exploring the normative case for international economic cooperation, as well as the obstacles to achieve it, it focuses in the achievements and challenges ahead for global economic governance in a number of economic issue areas. In order to do so, it analyzes the role of the G-20, an institution that has emerged since 2008 as the focal point for global economic coordination.
\end{abstract}

Keywords: Economic Governance, International Cooperation, Protectionism, Currency Wars, IMF.

Clasificación JEL: F50, F53, F55

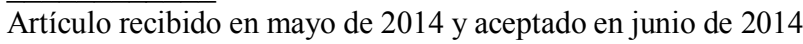

Artículo disponible en versión electrónica en la página www.revista-eea.net, ref. ə-32311 


\section{INTRODUCCIÓN}

Ya antes de la crisis financiera global que estalló en 2008 se vislumbraba la necesidad de potenciar las reglas económicas globales para que la interacción entre economías nacionales muy interconectadas con regulaciones esencialmente nacionales, segmentadas e inconexas, no generara resultados perversos. De hecho, en la primera década del siglo XXI, numerosos autores se mostraban relativamente optimistas sobre las perspectivas de mejora en la cooperación económica internacional y la gobernanza de la globalización (Dervis, 2005; Rodrik, 2000). A pesar de que el mundo se estaba volviendo económicamente más multipolar (lo que daba lugar a mayores conflictos), el crecimiento económico era generalizado, el aumento de la desigualdad no estaba en el debate público y la rivalidad entre grandes potencias parecía gestionable. Sin embargo, con la crisis financiera global y la Gran Recesión, se ha puesto de manifiesto que mejorar la cooperación económica internacional y dar respuestas globales a los principales retos de la globalización se ha vuelto imprescindible. La autoregulación de los mercados financieros no ha funcionado como se esperaba, los problemas vinculados al cambio climático no han hecho más que aumentar, las instituciones económicas internacionales continúan estando cuestionadas $\mathrm{y}$, en casos en los que se han producido avances (como por ejemplo en la reducción de los desequilibrios macroeconómicos globales) la cooperación internacional ha jugado un papel limitado.

Paradójicamente, mientras que tras el estallido de la crisis financiera global en 2008 se sucedieron importantes iniciativas de cooperación económica internacional en el marco del G-20 que permitieron evitar una segunda Gran Depresión y redujeron el impacto adverso de la crisis financiera, desde que en 2010 la sensación de riesgo de colapso sistémico se difuminara, los esfuerzos de cooperación económica internacional se han reducido. Además, han aumentado las dudas sobre la capacidad de la comunidad internacional de dotarse de mecanismos efectivos de gobernanza económica global (Roubini y Bremmer, 2011; Held y Roger, 2013). Goldin resume bien el sentir general en el mundo de los policy makers: "the stakes could not be higher. Unless we are able to more effectively manage the risks associated with globalization, they will overwhelm us. This is the core challenge of our times" (Goldin, 2013: 1). Sin embargo, como veremos, las perspectivas para la cooperación no son favorables.

Los intereses económicos nacionales, la resistencia de la mayoría de los Estados a ceder mayores cuotas de soberanía a instituciones supranacionales y las distintas interpretaciones de cuáles son las mejores políticas para superar la "resaca" de la Gran Recesión, mejorar la regulación económica o distribuir los costes de la lucha contra el cambio climático están dificultando la cooperación económica internacional y haciendo que el G-20 casi no genere avances. 
Por lo tanto, mejorar la gobernanza de la globalización económica sigue siendo una asignatura pendiente (Stiglitz y Kaldor, 2013). Es utópico pensar que será posible crear sistemas de gobernanza internacional democráticos y legítimos en todos los ámbitos relevantes. Además, la experiencia histórica indica que armonizar la legislación nacional de todos los países no es siempre una buena idea, tanto porque los distintos gobiernos no se ponen de acuerdo sobre cuál es el marco institucional y regulatorio más adecuado como porque el margen de maniobra para la innovación institucional es clave para el crecimiento de los países en desarrollo (y las reglas globales pueden dificultarlo (Rodrik, 2011)). Pero gobernanza económica global no tiene por qué significar reglas comunes en todos los ámbitos. De hecho, en los aspectos en los que no exista consenso sobre qué tipo de reglas supranacionales comunes adoptar habría que asegurarse de que los mercados no van más allá de los marcos regulatorios nacionales. Esta decisión de limitar parcialmente la globalización económica en algunos aspectos desestabilizadores (como por ejemplo regulando de forma distinta los movimientos internacionales de capital en función de las necesidades de cada país o incluso prohibiendo algunos instrumentos financieros) también debería ser consensuada y, por lo tanto, formar parte de lo que llamamos gobernanza económica global.

Este artículo analiza los actuales retos a los que se enfrenta la comunidad internacional para mejorar la gobernanza de los aspectos de la globalización económica que generaran mayores externalidades negativas transfronterizas y que requieren, por tanto, algún tipo de coordinación internacional. Tras plantear una justificación normativa de dónde y cuándo es necesaria esta gobernanza económica supranacional, así como cuáles son las dificultades para alcanzarla, se procede a explorar los avances y asignaturas pendientes en las distintas áreas económicas relevantes. Para ello, se analiza el papel del G-20, que desde 2008 ha emergido como en el epicentro de la gobernanza económica internacional.

\section{DÓNDE Y QUÉ GOBERNAR: JUSTIFICACIÓN NORMATIVA DE LA GOBERNANZA ECONÓMICA GLOBAL}

Se ha vuelto un cliché afirmar que los problemas globales requieren soluciones globales y que la globalización ofrece grandes oportunidades pero necesita ser gobernada (Goldin, 2013, Ocampo, 2010; Stiglitz, 2006). Sin embargo, como mostrara Keohane (1984) hace tres décadas, la cooperación internacional no es ni mucho menos necesaria para alcanzar resultados satisfactorios en todas las áreas de la economía política internacional.

La Figura 1 permite diferenciar entre las distintas formas de interacción entre países en el ámbito internacional. Estas relaciones pueden ser armónicas, cooperativas o conflictivas. La cooperación requiere de la existencia de un conflicto 
de intereses entre Estados y de la superación del conflicto mediante la negociación política. Es importante resaltar la diferencia entre la armonía y la cooperación, que son conceptos distintos. Como indica Keohane (1984:53): "La armonía es apolítica. No requiere de ninguna comunicación ni del ejercicio de influencia política alguna. Por el contrario, la cooperación es política: de alguna forma los patrones de comportamiento [de los países] deben ser modificados." Para que se produzca la cooperación es necesario que todos los actores tengan algo que ganar a través de la negociación, es decir, que no nos encontremos ante un juego de suma cero.

Figura 1

Armonía, cooperación y conflicto

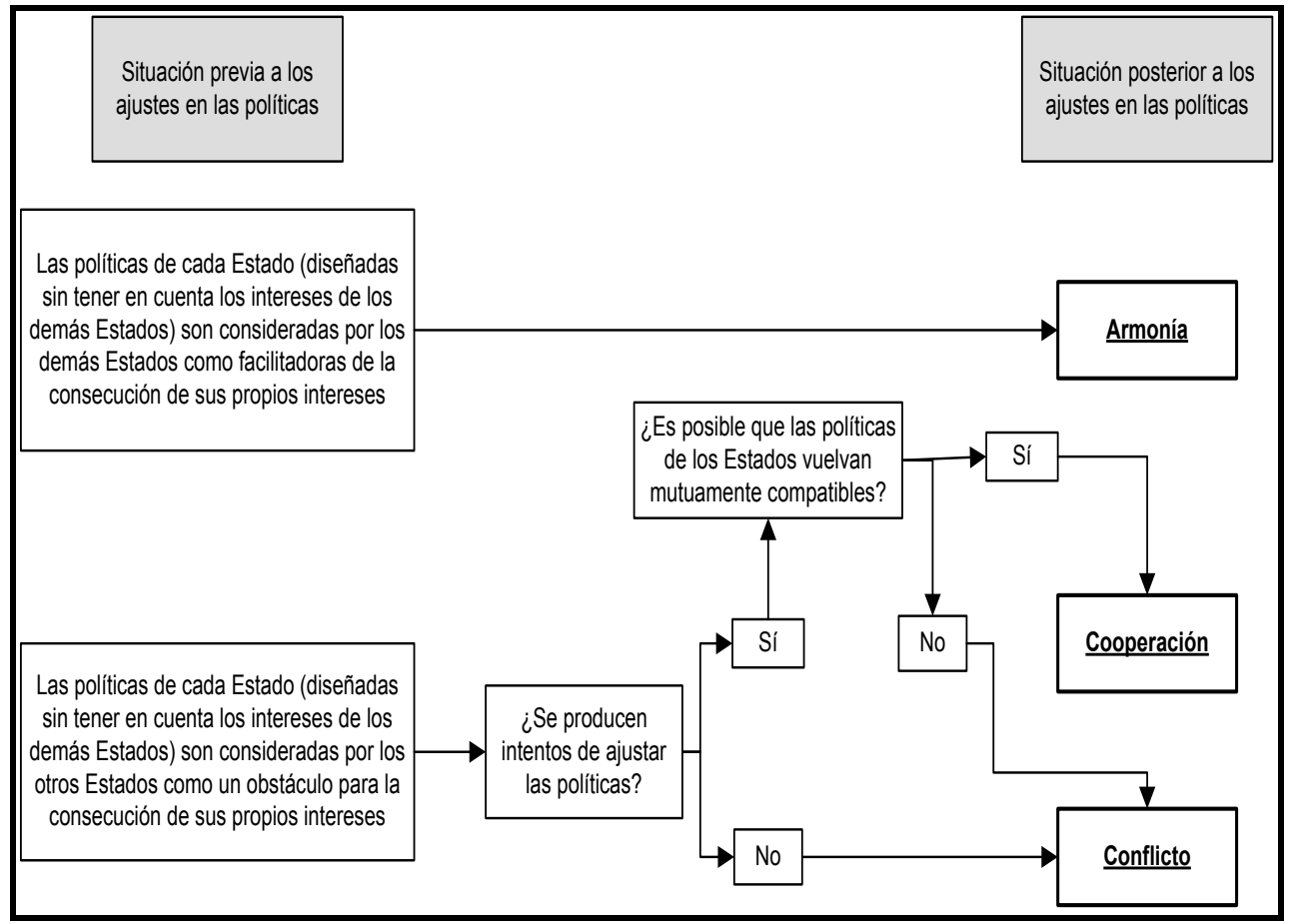

Fuente: Elaboración propia adaptado de Keohane (1984:53).

Aun aceptando la proposición central de la escuela realista de relaciones internacionales, que sostiene que los estados que integran un sistema anárquico intentarán aumentar su poder y riqueza actuando siempre de modo oportunista, en ocasiones es posible llegar a un acuerdo que beneficie a todos mediante la negociación si sus intereses son compatibles (ese es, de hecho, uno de los grandes postulados de la escuela liberal-institucionalista de las relaciones interna- 
cionales). Esta compatibilidad de intereses no siempre existe, lo que explica que la cooperación internacional no sea constante sino variable.

En todo caso, dada la dificultad que existe para alcanzar acuerdos internacionales, resulta conveniente dejar de lado por un momento el diseño de los mecanismos destinados a mejorar las posibilidades de cooperación en las principales áreas (del que nos ocuparemos más abajo) para examinar cuál es la justificación teórica para la cooperación. ${ }^{1}$

La justificación normativa para que distintos países coordinen sus políticas públicas se apoya de la teoría de los bienes públicos, en particular, en la de los bienes públicos globales (Kaul et al., 1999; Kaul et al., 2003). Los bienes (o males) públicos son aquellos no rivales en el consumo y no excluibles a través del sistema de precios (Samuelson, 1954). Cuando estos tienen carácter transfronterizo se habla de bienes públicos internacionales. El mejor ejemplo sería el cambio climático, donde cualquier país sufre los efectos de la volatilidad del clima generados por la acumulación de gases de efecto invernadero independientemente de las emisiones de los mismos que su economía produzca. En estos casos la cooperación internacional está plenamente justificada. En su ausencia se producirá una sub provisión de estos bienes (o una sobre producción en el caso de ser males, como la contaminación) que colocará a la comunidad internacional en una situación subóptima. Ahora bien, hay que reconocer que los bienes públicos globales puros son muy pocos, por lo que la cooperación no siempre estará justificada.

En el extremo opuesto a los bienes públicos globales se ubican las políticas económicas nacionales que no tienen ningún efecto transfronterizo directo. Sería el caso de las políticas educativas, laborales o de las de urbanismo. En estos casos no hay necesidad de coordinar las políticas de los distintos países, por lo que resulta eficiente (y además legítimo) que sean las autoridades nacionales las que las decidan sin interferencia externa. ${ }^{2}$

A medio camino entre las políticas sin implicaciones internacionales y los bienes públicos globales, a veces llamados global commons, es posible distinguir otras dos situaciones que también requieren de coordinación: las llamadas políticas de empobrecimiento del vecino, cuyo objetivo es precisamente obtener una ventaja económica a expensas de otros países; y las que Frieden et al. (2012) llaman políticas de auto-empobrecimiento, que también tiene efectos externos negativos aunque no sean intencionados.

\footnotetext{
${ }^{1}$ A continuación se sigue la tipología planteada en Frieden et al. (2012) para establecer qué tipo de ámbitos económicos internacionales requieren de cooperación internacional.

${ }^{2}$ En áreas económicas tan integradas como la zona euro, que cuentan con una moneda común, sí que existe una justificación para coordinar prácticamente todas las políticas económicas de los países, como de hecho prevé el Procedimiento de Desequilibrios Macroeconómicos aprobado en 2011 como parte del "six-pack" por la Unión Europea.
} 
En el primero de los casos nos encontramos ante las clásicas actuaciones en las que un país intenta "abusar" de su posición dominante para extraer beneficios económicos a expensas de los demás en un juego de suma cero. Se trata, por ejemplo, de la (rara) aplicación de la teoría del arancel óptimo para un país grande y con poder de mercado (según la cual el establecimiento de un arancel puede incrementar el bienestar nacional a expensas del de los socios comerciales (Irwin, 1996)) o de las devaluaciones competitivas (hoy rebautizadas como "guerras de divisas"), donde los países intentan promover el crecimiento fomentando las exportaciones a expensas de terceros países. En estos casos, que en su mayoría tienen una estructura clásica de "dilema del prisionero", la cooperación internacional aparece como necesaria para regular los efectos adversos transfronterizos de las políticas nacionales. Esto es especialmente importante ya que, en ausencia de coordinación, las políticas de empobrecer al vecino pueden desencadenar conflictos de mayor calado (el proteccionismo como resultado de las devaluaciones competitivas durante los años treinta sería el ejemplo clásico).

En el segundo de los casos, mucho menos estudiado en la literatura, nos encontramos ante políticas cuyo objetivo es doméstico pero que, además, tienen efectos externos no intencionados sobre terceros países, además de ser perjudiciales para el país que las aplica (de ahí el nombre de "políticas de auto-empobrecimiento"). Un ejemplo serían los subsidios agrícolas en la Unión Europea, que, además de ser perjudiciales para la mayoría de los ciudadanos europeos, tienen efectos internacionales al reducir los precios agrícolas mundiales, lo que perjudica a los exportadores de alimentos y beneficia a los importadores. Otro ejemplo, de mayor actualidad, sería la liberalización financiera estadounidense que precedió a la crisis financiera global que estalló en 2008, que vista en perspectiva fue una política dañina para Estados Unidos, pero también para el resto del mundo. Lo importante de estas políticas es que tanto su objetivo como sus efectos son fundamentalmente internos (en la mayoría de los casos redistributivos hacia grupos de presión concretos que pueden determinar la legislación), pero terminan generando externalidades que necesitan ser internalizadas mediante la coordinación internacional. En estos casos, se hace necesario establecer regímenes internacionales que mejoren el mecanismo de toma de decisiones a nivel nacional, así como reglas para mejorar la transparencia, la rendición de cuentas o la representatividad.

En definitiva, la justificación normativa para la gobernanza global es clara, pero los instrumentos que deben adoptarse varían de caso a caso. En algunos habrá que integrar las políticas nacionales en acuerdos supranacionales para evitar la generación de males públicos globales. En otros habrá que coordinar las políticas para internalizar las externalidades negativas de las políticas nacionales. Finalmente, también hay casos en los que será necesario utilizar la cooperación para mejorar el mecanismo de toma de decisiones al nivel nacional. 


\section{POR QUÉ ES TAN DIFÍCIL LA GOBERNANZA GLOBAL}

Más allá de la justificación normativa para la cooperación internacional, lo cierto es que, en la práctica, según indica Cohen (2000:246): "la cooperación internacional, como el amor pasional, es algo bueno pero difícil de sostener". Y ello se debe a que la cooperación exige adaptar las políticas económicas nacionales a las necesidades del sistema (y en ocasiones de otros países) y, en los casos de "cooperación fuerte", entrar en acuerdos internacionales que implican una cesión de soberanía a instancias supranacionales. Esta cesión permanente de soberanía, que resulta mucho menos reversible que la mera coordinación puntual de políticas, es lo que la literatura identifica como la creación de regímenes internacionales (Krasner, 1983) que configuran la gobernanza económica global. Así, para Lamy (2008), la gobernanza de la globalización debe ser entendida no como un gobierno global, sino como un procedimiento de toma de decisiones basado en la negociación permanente y el respeto a la ley. De forma similar, Frieden (2012: 3) indica que "Governance is taken to mean the authoritative provision of government-like functions; there is some decision rule by which choices are made, and some way of enforcing rules".

Ahora bien, la gobernanza global puede ser o no democrática y/o legítima. Como señala Keohane (2003: 3): "Global governance refers to the creation of rules and the exercise of power on a global scale [...] Since there is no global government, global governance entails strategic interaction among entities that are not hierarchically distributed". Por lo tanto, la gobernanza global puede ejercerse desde distintos foros que pueden o no contar con la legitimidad necesaria y que pueden no operar de forma transparente.

Aun así, la comunidad internacional ha intentado establecer mecanismos institucionales (formales o informales) que emanen de la negociación intergubernamental y que aspiren a ser concebidos como legítimos (además de resultar efectivos) para las principales potencias del sistema. ${ }^{3}$ En momentos como el actual, donde no existe una potencia económica hegemónica capaz de imponer sus preferencias, ello pasa por establecer (o modernizar) regímenes internacionales capaces de ejercer la gobernanza global en áreas determinadas de la economía internacional donde se justifique la coordinación de políticas.

Así, "los regímenes pueden ser definidos como un conjunto implícito o explícito de principios, normas, reglas y procedimientos de toma de decisión alrededor de los cuales las expectativas de los distintos actores convergen en un área determinada de las relaciones internacionales" (Krasner, 1983:2). Aparecen alrededor de temas concretos. No especifican necesariamente reglas de coopera-

\footnotetext{
${ }^{3}$ En este sentido, hay incluso propuestas para incorporar a la sociedad civil transancional a los mecanismos de gobernanza económica global, de forma que su legitimidad pueda aumentar. Véase, por ejemplo Anheier (2013).
} 
ción per se, sino que describen áreas específicas de gobierno colectivo en temas de interés para la comunidad internacional, que van desde la gestión de los sistemas monetario o comercial internacionales hasta las normas de protección del medio ambiente, la regulación de los flujos migratorios o los marcos que gobiernan las políticas de cooperación al desarrollo.

Los regímenes suelen tomar la forma de organizaciones internacionales y acostumbran a tener sedes permanentes, como la Organización Mundial del Comercio (OMC), el Fondo Monetario Internacional (FMI), el Banco Mundial o la Organización para la Cooperación y el Desarrollo Económico (OCDE). Sin embargo, también pueden existir como una serie de cumbres intergubernamentales que se repiten a lo largo del tiempo, como el G-7/8, el G-20, las reuniones sobre desarrollo sostenible impulsadas por las Naciones Unidas o las cumbres de la Organización de los Países Exportadores de Petróleo (OPEP).

Para que un acuerdo puntual se convierta en un regimen internacional, sus miembros deben reconocer la validez de sus acuerdos a lo largo del tiempo, así como dedicar recursos a mantenerlo. Los regímenes dan lugar a la institucionalización de la cooperación y permiten a los países gestionar conflictos derivados de la interdependencia en lugar de recurrir a estrategias unilaterales, incluso cuando estas estrategias pueden reportarles mayores beneficios en el corto plazo.

La función principal de los regímenes es, por lo tanto, la de facilitar acuerdos. Además, reducen los costes de transacción al establecer redes de comunicación mediante el establecimiento de instituciones formales, lo que reduce los costes de la negociación al permitir a los diplomáticos un rápido acceso a normas previamente aceptadas en materias conflictivas (Bayne y Woolcock, 2003). Ello evita que se vean involucrados en discusiones interminables y permite reducir el recurso a las amenazas para alcanzar acuerdos. Este es, por ejemplo, el fundamento teórico del mecanismo de resolución de conflictos que se incorporó en la OMC en 1995. Este mecanismo facilita el cumplimiento de las normas multilaterales del comercio internacional en casos de conflicto mediante una secuencia de reglas que culmina en un arbitraje de obligado cumplimiento decidido por un panel de expertos independientes.

Por otra parte, los regímenes sirven para aumentar la información y reducir la incertidumbre. Permiten organizar y estructurar la interacción repetida entre países al establecer de modo estable unas reglas de comportamiento compartidas, que permiten a los negociadores anticipar el comportamiento de sus colegas de otras nacionalidades. Asimismo, una vez que se ha establecido un régimen y que han entrado en vigor varios acuerdos, se reducirán los incentivos para que los Estados violen sus compromisos, al tiempo que aumentarán los beneficios esperados de seguir operando dentro del procedimiento adoptado. Este proceso permite aumentar la información disponible e incrementar la vigilancia de unos 
Estados sobre otros. Por último, los regímenes permiten la negociación simultánea sobre diversos temas interrelacionados, lo que facilita la formación de coaliciones entre distintos países con los mismos intereses, así como el uso de "pagos compensatorios" que facilitan los acuerdos.

El problema es que, como señala Keohane (1984: 50), es más fácil mantener un régimen que crearlo, sobre todo en ausencia de potencias hegemónicas con claro dominio sobre el resto. Y, en la actualidad, el sistema económico internacional está en transición (Zakaria, 2008; Banco Mundial, 2011): el auge de las potencias emergentes y el declive relativo de Occidente están haciendo que muchos de los regímenes e instituciones de gobernanza económica global que sirvieron en el pasado se hayan quedado obsoletos, pero aún no han aparecido otros capaces de sustituirlos de forma efectiva, y la adaptación de los existentes a las nuevas realidades geoeconómicas está siendo lenta.

Como señala Cohen (2013: 46) la cooperación económica internacional en momentos de transición como el actual requiere al menos de dos condiciones. En primer lugar, unos valores medianamente compartidos por los principales países sobre qué políticas son necesarias. Y en segundo, un liderazgo capaz de catalizar esos valores en acciones concretas. Ninguna de las dos se cumple en la actualidad, algo que sí ocurrió, por ejemplo, al final de la Segunda Guerra Mundial, lo que dificulta enormemente la cooperación. Como indica Helleiner (2010: 619, 636): "The success of the Bretton Woods conference [in 1944] was a product of a remarkable combination of concentrated power in the state system [and] a transnational expert consensus.... The political conditions that generated the innovations of Bretton Woods were unique and are not present today." Hoy, los países emergentes tienen visiones sobre la regulación financiera, los mecanismos de ajuste en el sistema monetario internacional o las mejores prácticas en política comercial que son muy distintas a las de los países avanzados. Incluso tienen una visión sobre cómo debería funcionar (y regularse) el capitalismo que dista mucho de la dominante en Washington o Londres (Ban y Blyth, 2013). Además, tras décadas de liderazgo intelectual y político anglo sajón en asuntos económicos internacionales, la crisis financiera global ha supuesto un importante golpe a la visión norteamericana del mercado, lo que ha puesto a Estados Unidos a la defensiva, generando un vació de poder significativo en el sistema internacional, que ni los países emergentes ni la Unión Europea están dispuestos a ocupar. Esto explicaría las enormes dificultades que está teniendo la comunidad internacional para establecer nuevos sistemas de gobernanza económica global efectiva, así como las malas perspectivas para que esta situación pueda cambiar en el futuro próximo.

Si bien es cierto que a finales de 2008 se produjo una acción concertada de los gobiernos en el seno del G-20 para evitar el colapso del sistema financiero internacional tras la quiebra de Lehman Brothers, y que esta coordinación con- 
tinuó durante 2009 con planes de expansión fiscal en los principales países, desde entonces la cooperación internacional prácticamente ha desaparecido. Como subraya Cohen (2013), en realidad, estos episodios de cooperación no requirieron demasiados ajustes en las políticas nacionales, ya que todos los países hubieran optado por proteger sus sistemas financieros de la quiebra e impulsar estímulos monetarios y fiscales incluso en ausencia de coordinación. Por lo tanto, lo que ocurrió en 2008-2009 más bien correspondería (dentro de la tipología explicada arriba) a una situación de armonía entre actuaciones estatales unilaterales con un mínimo de coordinación, y no a un ejercicio de auténtica cooperación internacional. De haberlo sido, las políticas de los principales países tendrían que haberse ajustado a las de los demás para generar un resultado óptimo desde el punto de vista global.

Aun así, lo que logró la comunidad internacional fue ubicar al G-20, al menos formalmente, como el epicentro de la gobernanza económica global. A continuación analizamos en mayor detalle ese proceso, así como qué ha conseguido y que tiene pendiente lograr este nuevo (y emergente) régimen internacional. Al referirnos a los logros y las asignaturas pendientes del G-20, analizaremos los temas en los que ha logrado avances concretos o en los que ha intentado poner las bases para futuros acuerdos. No pretendemos hacer un tratamiento exhaustivo de todos los temas que conforman la agenda de la gobernanza económica global porque ello exigiría un análisis mucho más extenso, que además trascendería a los temas económicos. ${ }^{4}$

\section{LA GOBERNANZA ECONÓMICA GLOBAL EN LA PRÁCTICA: LUCES Y SOMBRAS DEL G-20}

Como se ha señalado arriba, al estallar la crisis financiera global se produjo un importante esfuerzo de coordinación económica internacional a través de las cumbres del G-20 de Washington (noviembre de 2008) y Londres (abril de 2009), que puede considerarse como relativamente exitoso. Contribuyó a evitar una quiebra del sistema de pagos internacional, suavizó el colapso de la producción mundial, y ayudó a mantener a raya el proteccionismo. Es por ello que suele decirse que la respuesta política a la crisis financiera global, coordinada por el G-20, sirvió para evitar una segunda Gran Depresión (Steinberg, 2011).

El hecho de que fuera el G-20 y no el G-7/8 quien coordinara la respuesta internacional a la crisis supuso un paso adelante en el aumento de legitimidad de los foros de decisión económica internacionales porque el G-20 tiene una nutrida representación de países emergentes, que venían años demandando una

\footnotetext{
${ }^{4}$ Para un análisis más exhaustivo de la amplísima amalgama de temas que incluye la gobernanza global véase Held y Roger (2013); Stiglitz y Kaldor (2013); Frieden et al. (2012) o Goldin (2013).
} 
mayor voz en los organismos de decisión internacionales. Esto llevó a algunos analistas a llegar a hablar de un nuevo "momento Bretton Woods" que significaría el comienzo de la nueva gobernanza económica global que necesitaba la nueva realidad geo-económica del siglo XXI (Helleiner y Pagliari, 2009; Garret, 2010).

Este optimismo inicial sobre el G-20 se basó en que aparecía como un foro suficientemente amplio (y por tanto legítimo) como para convertirse en el embrión de ciertas reformas económicas globales consensuadas, al tiempo que era lo suficientemente pequeño como para ser efectivo. ${ }^{5} \mathrm{Su}$ principal virtud era (y sigue siendo) que cuenta con un buen equilibrio entre países avanzados y emergentes, algo que no sucede con prácticamente ninguno de los grupos " $G$ " del sistema internacional donde se practica y ejerce el "minilateralismo" de forma efectiva.

Pero el G-20 no es una institución internacional ni cuenta con un secretariado permanente o con recursos propios. Tampoco está formalmente integrado en el sistema de Naciones Unidas y su membresía ha sido criticada por no tener suficientes equilibrios geográficos o por tomar decisiones que afectan a 175 países que no son miembros del Club (Woods, 2013: 348). Organiza su actividad en torno a las iniciativas de la presidencia de turno a través de grupos de trabajo ad hoc establecidos para los distintos temas de su agenda. La informalidad y los encuentros cara a cara entre Jefes de Estado y de Gobierno le otorgan flexibilidad (sobre todo comparado con las complejas burocracias de las instituciones formales), facilitando la toma de decisiones. Sin embargo, como no es una institución internacional, no puede obligar a ningún país a que cumpla sus acuerdos y tampoco puede establecer sanciones o incentivos de forma multilateral. Esta falta tanto de estructura como de institucionalidad se ve parcialmente compensada porque sus trabajos se apoyan (e influyen) en otras instituciones económicas internacionales que sí cuentan con el suficiente apoyo técnico y financiero y que sí tienen cierta capacidad de coerción sobre sus estados miembros (principalmente el FMI, Consejo de Estabilidad Financiera (CEF), el Banco Mundial y la OMC).

Por lo tanto, aunque desde la Cumbre de Pittsburgh de 2011, el G-20 se auto-declaró el epicentro de la gobernanza económica global, está aún muy lejos de serlo. De hecho, sólo puede actuar como punto focal para el diseño de nuevas reglas que coordinen tanto las reformas de la legislación económica interna de

\footnotetext{
${ }^{5}$ Los miembros del G-20 son Alemania, Canadá, Estados Unidos, Francia, Italia, Japón, Reino Unido y Rusia (G-8), Arabia Saudita, Argentina, Australia, Brasil, China , India, Indonesia, México, República de Corea, Sudáfrica, Turquía y la Unión Europea. Desde 2010 España es invitado permanente. El grupo, creado en 1997 tras la crisis financiera asiática, pasó a reunirse al nivel de Jefes de Estado y de Gobierno en Noviembre de 2008. Anteriormente, lo hacía al nivel de Ministros de Economía y Finanzas.
} 
los países como los estatutos de las organizaciones económicas internacionales para adaptarlos a la nueva realidad de la globalización. Asimismo, sigue siendo foco de críticas por aquellos que consideran que su legitimidad y representatividad no son suficientes.

Como se ha señalado, sus avances a partir de 2010 están siendo lentos, los conflictos en las reuniones de alto nivel han aumentado y la influencia de los países emergentes no ha aumentado demasiado en relación a los viejos tiempos del G-7 (Woods, 2013). Las grandes potencias han dado prioridad a sus problemas económicos internos sobre la resolución de los problemas transnacionales: la Unión Europea se ha volcado en resolver la crisis de la zona euro, Estados Unidos en combatir el elevado desempleo y reformar su sistema de salud y los países emergentes en evitar el sobrecalentamiento de sus economías (en un primer momento) y evitar su desaceleración (a partir de la retirada de estímulos monetarios de la Fed en 2013). Además, las reuniones del G-20, como ya ocurriera con las del G-7/8, se han centrado en problemas políticos coyunturales de carácter urgente (como la crisis en Siria en la Cumbre del G-20 San Petersburgo en 2013), lo que ha desplazado la atención de los problemas importantes de largo plazo. De hecho, a partir de 2010, además de un parón en cooperación económica internacional han aparecido nuevos conflictos, como la "guerra de divisas" (que comenzó en 2010 y ha vuelto a intensificarse en 2013), las tensiones en los mercados energéticos y alimentarios o la imposibilidad de cerrar un acuerdo post-Kioto en las negociaciones sobre cambio climático, concluir la Ronda de Doha de la OMC o avanzar con celeridad en la reforma del sistema monetario internacional.

Aun así, como subraya Kirton (2013), se han producido ciertos avances y se ha mejorado sensiblemente el marco institucional para que las principales potencias tengan un diálogo continuado, que es la única forma de que se vayan produciendo cambios graduales. El G-20 no es perfecto, pero es un foro mucho mejor que sus antecesores para ejercer el liderazgo compartido que requiere una economía multipolar. Aunque le faltan algunos países y le sobran otros, lo integran un número de estados lo suficientemente grande como para tener legitimidad y lo suficientemente pequeño como para poder ser efectivo. En todo caso, este grupo no debe confundirse con un nuevo gobierno económico mundial, sino que, en el mejor de los casos, servirá como un catalizador de las decisiones que después deben plasmarse en instituciones como el FMI o la OMC. A continuación analizamos brevemente sus principales luces y sombras. ${ }^{6}$

\footnotetext{
${ }^{6}$ Para un análisis detallado de todas las iniciativas tomadas por el G-20, así como su grado de cumplimiento, el grupo de investigación sobre el G20 de la Universidad de Toronto dirigido por el profesor Kirton se ha convertido en la referencia mundial (http://www.g20.utoronto.ca/). Véase también Kirton (2013).
} 


\section{PEQUEÑOS AVANCES Y ASIGNATURAS PENDIENTES}

Como la deficiente regulación financiera había sido una de las causas principales de la crisis financiera global, desde la cumbre del G-20 en Washington en 2008, se forjó cierto consenso sobre la necesidad de limitar los niveles de apalancamiento financiero y riesgo, aumentar la información y la transparencia en los mercados financieros, cambiar los incentivos y la forma de remunerar a los ejecutivos del sector, redefinir y homogeneizar las reglas de valoración contable, aumentar los requerimientos de capital de las instituciones financieras, extender la regulación a algunos mercados hasta entonces opacos y supervisar mejor los mercados de derivados. En todos estos aspectos, tanto el G-20 como el Consejo de Estabilidad Financiera (que sustituyó al Foro de Estabilidad Financiera en la cumbre de Londres del G-20 y se amplió a los países emergentes y a España) actuaron como centros de coordinación de las reformas adoptadas al nivel nacional. También sirvieron para promover la adopción de los criterios de Basilea III por los que se aumentará el capital "de alta calidad" de las instituciones financieras a partir de 2018.

En todo caso, hay que señalar que los principales avances en materia de reforma financiera han respondido a las propias iniciativas reguladoras en los países occidentales (sobre todo en Estados Unidos y la Unión Europea) y no al G-20 en sí, y que en muchos de los aspectos clave no se han podido más que adoptar códigos de conducta genéricos que no han reformado significativamente el sistema (Eichengreen, 2013). No se ha acordado nada parecido a unas reglas financieras globales más allá de lo pactado en Basilea III, ni se ha abordado el problema de las instituciones sistémicas (too big to fail). Tampoco se han tomado medidas para cambiar el comportamiento de las agencias de calificación, acabar con los paraísos fiscales o establecer un impuesto global sobre las transacciones financieras, que sí se ha puesto en macha en 11 países de la Unión Europea. También cabe señalar que la reforma financiera en Estados Unidos ha sido bastante más tímida de lo que en un principio se pensó, sobre todo debido a la presión del lobby financiero.

Otro tema en el que se han producido ciertos avances es el de los desequilibrios macroeconómicos globales (exceso de ahorro en China, Japón, Alemania, los países exportadores de petróleo y algunas otras potencias emergentes asiáticas y exceso de gasto en Estados Unidos, Reino Unido y los países periféricos de la Unión Europea). Esta creciente divergencia entre ahorro y gasto nacional generaba desequilibrios externos y una excesiva acumulación de reservas en países con superávit por cuenta corriente -sobre todo si tenían tipos de cambio fijos-, así como tensiones en los mercados cambiarios, que amenazaban con desencadenar guerras comerciales. Además, este modelo de crecimiento tan desequilibrado, que se ha bautizado como Bretton Woods II, fue uno de los causantes de la crisis (Wolf, 2009; Roubini y Mihm, 2010). Por ello era importante 
reequilibrar la economía mundial y reformar el sistema monetario internacional, lo que requería que China apreciara su moneda y la desligara del dólar, Estados Unidos aumentara su tasa de ahorro y Japón y la Unión Europea incrementaran su demanda interna. El "Marco para el crecimiento sostenible y equilibrado" aprobado por el G-20 estaba pensado precisamente para facilitar este proceso de reequilibrio bajo la supervisión del FMI.

Sin embargo, en la práctica, los desequilibrios macroeconómicos globales se han moderado no por la actuación del G-20, sino por la propia dinámica de la recesión global (Eichengreen, 2014), que redujo la demanda interna de Estados Unidos (y con ella su déficit por cuenta corriente) al tiempo que forzaba a China a iniciar una estrategia para reequilibrar su economía desde el ahorro, la inversión y las exportaciones hacia un mayor consumo interno y una expansión del sector servicios. La zona euro, por su parte, con sus políticas de austeridad, ha pasado de estar en equilibrio externo a tener un superávit por cuenta corriente que comienza a preocupar al ser un factor deflacionista en la economía mundial.

Por otra parte, pero también en el campo macroeconómico, desde 2010, el G-20 se ha convertido en el foro en el que se ha escenificado el conflicto entre Estados Unidos, la Unión Europea y los países emergentes sobre qué curso debía seguir la política macroeconómica a nivel global tras los estímulos acordados en 2008 y 2009. Los países europeos, liderados por Alemania, abogaron por la austeridad fiscal en el contexto de la crisis del euro, mientras que la Administración Obama defendió la continuidad de los estímulos, tanto fiscales como monetarios. Asimismo, las políticas de expansión cuantitativa de la Fed, abrieron un conflicto entre Estados Unidos y los países emergentes, que sin llegar a desencadenar una crisis, tensó de forma significativa el hasta entonces buen diálogo macroeconómico en el G-20 abriendo las conocidas como guerras de divisas (Steinberg, 2013). A pesar de los esfuerzos en las cumbres de Seúl de 2010 y de Cannes de 2011 por atajar estos problemas, no se alcanzó ningún acuerdo ni para coordinar las políticas macroeconómicas ni para reformar el sistema monetario internacional.

En este último tema, el del desgobierno del sistema monetario internacional, la comunidad internacional se enfrenta a un problema grave (Cohen 2013). El sistema sigue girando en torno al dólar, pero Estados Unidos ya no es la superpotencia hegemónica del pasado, y sigue explotando de forma oportunista el privilegio de ser el emisor de la moneda de reserva mundial (Eichengreen, 2011). Por ello, sería necesario que el sistema internacional fuese capaz de generar otros activos de reserva globales que funcionaran como fuentes de liquidez, dieran una mayor estabilidad al sistema y sirvieran para reflejar al nivel monetario y político los cambios que la economía mundial ha experimentado en las últimas décadas. Sin embargo, como por el momento el euro no es capaz de rivalizar con el dólar y el yuan chino todavía no es convertible, la economía 
mundial sigue dependiendo del dólar, por lo que la política monetaria de Estados Unidos causa externalidades negativas a los demás países. Aunque la presidencia francesa del G-20 de 2011 incluyó este tema en la agenda, y China y otros países emergentes lo apoyaron con entusiasmo, al final no fue posible alcanzar ningún acuerdo.

El último tema en el que el G20 ha logrado algunos avances es el de la reforma del FMI. Tras años de irrelevancia, el FMI ha mantenido una importante actividad desde 2008, acudiendo al rescate de países con necesidades de liquidez mediante nuevos instrumentos como la línea de crédito flexible o la línea de crédito precautorio y teniendo una participación activa en la crisis del euro desde 2009. Además, contribuyó a coordinar el estímulo fiscal global impulsado por el G-20 en 2009 y logró cuadruplicar su financiación hasta alcanzar el billón de dólares (Moreno, 2013). Asimismo, el G-20 acordó en 2010 una reforma de la gobernanza interna del FMI. Pactó trasladar un $6 \%$ de las cuotas (y, por tanto, un porcentaje de votos similar) desde los países avanzados a los emergentes. Este ajuste, que se suma a la reforma de cuotas y votos adoptada entre 2006 y 2008, llevará a China desde la sexta a la tercera posición, quedando sólo por detrás de Estados Unidos y Japón en número de votos en la institución. Además, en una histórica decisión, los países europeos acordaron ceder dos de sus ocho sillas en la Junta Directiva del FMI (sobre un total de 24) a los países emergentes. A mediados de 2014, este acuerdo se encontraba bloqueado por el Congreso estadounidense, pero es de suponer que podrá salir adelante. De no hacerlo, supondría un serio revés para la institución (Truman, 2014).

El principal asunto en el que el G-20 ha intentado avanzar sin éxito es el cierre de la Ronda de Doha de la OMC, que sería esencial para consolidar a esta institución como punto central de la gobernanza del comercio mundial. Aunque la Gran Recesión no ha dado lugar a un auge del proteccionismo, el G-20 ha tenido un papel poco efectivo en asuntos comerciales. A pesar de haber reiterado en múltiples ocasiones la necesidad de cerrar la Ronda de Doha (abierta desde 2001), los enfrentamientos entre los países industrializados y los emergentes sobre el proteccionismo en materia agrícola y manufacturera han hecho imposible alcanzar un acuerdo. De hecho, desde 2008, muchos países han elevado sus aranceles, aunque siempre dentro de los límites establecidos por la OMC. También han introducido nuevas barreras no arancelarias, sobre todo en los ámbitos en los que la regulación de la OMC tiene más zonas grises, como las compras públicas o el apoyo a los sectores financieros o automovilísticos nacionales. Para consolidarse y evitar caer en la irrelevancia, la OMC necesita mostrarse ante la opinión pública internacional como la mejor opción para regular el comercio mundial. En los últimos años está viendo socavado su papel por un sinfín de iniciativas comerciales de carácter bilateral y regional. De hecho, el comercio internacional ha sufrido una transformación radical en las últimas décadas a la que la OMC está teniendo ciertos problemas para adaptarse 
(Baldwin, 2012). Han aparecido nuevas cadenas de suministro globales que permiten a las empresas multinacionales ubicar distintas partes del proceso productivo en distintos países para aprovechar las ventajas de costes. Sin embargo, estas cadenas de suministro requieren un nuevo tipo de reglas comerciales, centradas en la protección de los servicios o en la seguridad jurídica para las inversiones, aspectos en los que la OMC no ha sido capaz de establecer reglas globales. Sería deseable que la OMC pudiera dejar atrás la Ronda de Doha (bien cerrándola con un acuerdo de mínimos, bien declarándola muerta) y pasara a ocuparse de los temas comerciales relevantes para el siglo XXI, cómo multilateralizar la regulación comercial sobre cadenas de suministro globales, abordar los retos de la seguridad alimentaria y energética, o atreverse a entrar en ámbitos como la relación entre comercio y cambio climático, la manipulación de los tipos de cambio o la regulación de los fondos soberanos, que son los temas que interesan hoy a ciudadanos, empresas y gobiernos. De no hacerlo, su centralidad en el sistema comercial global se verá cada vez más amenazada por los nuevos grandes acuerdos comerciales preferenciales, en especial el TTIP entre la Unión Europea y Estados Unidos y el TPP entre los países de la Cuenca del Pacífico.

Por último, es necesario mencionar que el G-20 tiene como asignaturas pendientes adoptar iniciativas en la lucha contra la pobreza, el cambio climático y las migraciones internacionales. La aceptación del llamado "Consenso de Seúl" sobre el desarrollo adoptado por el G-20 en 2010 supone una dura crítica a las políticas de desarrollo del "Consenso de Washington", promulgadas por los países occidentales, el FMI y el Banco Mundial en los años noventa (Ban y Blyth 2013). Pero más allá de esta nueva declaración, que niega la validez de las recetas universales para el desarrollo y vuelve a dar al Estado un mayor papel en el proceso de crecimiento de los países pobres, no hay demasiado contenido en cuanto a nuevas fuentes de financiación al desarrollo o ayuda para los países menos avanzados (Ocampo, 2010). Asimismo, en los asuntos vinculados al Cambio Climático, los países emergentes se han negado a que el G-20 aborde la fijación de límites a las emisiones de gases de efecto invernadero porque en las Naciones Unidas tienen una posición negociadora más fuerte. Aun así, alcanzar algún tipo de acuerdo sobre este tema en el G- 20 sería enormemente útil porque dentro del grupo están los países responsables de más del $80 \%$ de las emisiones de gases de efecto invernadero. En el tema migratorio no ha habido ninguna propuesta, pero será un aspecto que irá ganando importancia en el futuro y que debería tener cierto marco regulatorio nacional, posiblemente dentro del sistema de Naciones Unidas.

\section{CONCLUSIÓN}

A lo largo de este trabajo se ha expuesto la necesidad de que la comunidad internacional avance en la configuración de un marco de gobernanza económica 
global capaz de dar respuesta a los crecientes retos económicos que plantean tanto la creciente interdependencia económica como los nuevos equilibrios de poder.

Por una parte se ha explicado por qué la justificación para la cooperación económica internacional es, en ocasiones, algo más compleja de lo que suele pensarse. Se ha insistido en que distintos ámbitos de la actividad económica transnacional requieren grados y fórmulas de regulación distintas, así como que la justificación para la homogeneización de regulaciones nacionales en todos los aspectos es bastante discutible.

A continuación se han explorado las razones por las que la cooperación económica internacional se ha vuelto tan compleja y difícil de conseguir. Se ha subrayado que la falta de un liderazgo claro, sumado a las visiones enfrentadas sobre las fórmulas para regular el capitalismo global que tienen las principales potencias, son los principales obstáculos para alcanzar acuerdos.

Por último, se ha procedido a evaluar el papel del G-20 como epicentro de la nueva gobernanza económica global. Se ha subrayado que desde su momento fundacional "estelar" en 2008-2009, el grupo ha ido perdiendo cohesión e impulso, tanto porque el posible colapso de la economía global ya no parecía un riesgo probable como porque las agendas políticas internas de los principales países restaban posibilidades a la cooperación internacional. Por lo tanto, el principal reto al que se enfrentará el G-20 es el de poder seguir siendo relevante. Para ello, tiene que demostrar que es útil en un contexto en el que la resaca de la Gran Recesión hace que muchos países estén dejando en segundo plano la cooperación internacional para dedicarse únicamente a sus prioridades de política interna.

\section{REFERENCIAS BIBLIOGRÁFICAS}

ANHEIER, HELMUT K. (2013). "Institutional Voids and the Role of Civil Society: the Case of Global Finance", Global Policy, Volume 5, Issue 1, pages 23-35,

BALDWIN, RICHARD (2012). "WTO 2.0: Global governance of supply-chain trade," Centre for Economic Policy Research Policy Insight number 64, Diciembre 2012.

BAN, CORNEL Y MARK BLYTH (2013). "The BRICs and the Washington Consensus: An introduction", Review of International Political Economy, 20:2, 241-255.

BANCO MUNDIAL (2011): Global Development Horizons 2011: Multipolarity - The New Global Economy. Washington DC.

BAYNE, NICHOLAS y STEPHEN WOOLCOCK (2003). The New Economic Diplomacy: decision making and negotiation in international economic relations. London: Ashgate.

BREMMER, IAN y NOURIEL. ROUBINI (2011). "A G-Zero World”, Foreign Affairs. March/April. 
COHEN, BENJAMIN (2000). "The triad and the unholy trinity: problems of international monetary cooperation" en Jeffrey Frieden y David Lake (editores): International Political Economy. Londres y Nueva York Bedford, St Martin's 2000, pp. 245-256.

COHEN, BENJAMIN (2013). "The Coming Global Monetary (Dis)Order", en David Held y Charles Roger (eds.), Global Governance at Risk. Polity Press.

DERVIS, KEMAL (2005): A Better Globalization: Legitimacy, Governance, and Reform. Center for Global Development. Washington DC.

EICHENGREEN, BARRY (2011). Exorbitant Privilege: The Rise and Fall of the Dollar, Oxford and New York, Oxford University Press.

EICHENGREEN, BARRY (2013): "Why No Glass-Steagall II?, Project Syndicate, 10 de enero de 2013.

EICHENGREEN, BARRY (2014). "A Requiem for Global Imbalances”, Project Syndicate, 13 de enero de 2014.

FRIEDEN, JEFFREY (2009). "The Crisis and beyond: Prospects for international economic cooperation" Policy Paper No. 5, Politics, Economics and Global Governance: The European Dimensions (London, December 2009).

FRIEDEN, JEFFREY (2012). "Global Economic Governance After the Crisis" Perspektiven der Wirtschaftspolitik 13. Pg. 1-12. http://scholar.harvard.edu/files/jfrieden/files/ global_economic_governance_after_the_crisis.pdf

FRIEDEN, JEFFREY, MICHAEL PETTIS, DANI RODRIK y ERNESTO ZEDILLO (2012). After the Fall: The Future of Global Cooperation. Geneva Reports on the World Economy 14.

GOLDIN, IAN (2013). Divided Nations. Oxford University Press.

GARRET, G. (2010). "G2 in G20: China, the United States and the World after the Global Financial Crisis". Global Policy, 1: 29-39.

HELD, DAVID y CHARLES ROGER (editors) (2013). Global Governance at Risk. Polity Press.

HELLEINER, ERIC (2010). "A Bretton Woods moment? The 2007-2008 crisis and the future of global finance", International Affairs 86(3), pp. 619-636.

HELLEINER, ERIC y STEFANO PAGLIARI (2009). "Towards a new Bretton Woods. The First G20 Leaders Sumit and the Regulation of Global Finance" New Political Economy, 14(2): 275-287.

IRWIN, DOUGLASS (1996). Against the Tide: An Intellectual History of Free Trade. Princeton University Press.

KAUL, INGE; GRUNBERG ISABELLE y MARC STEIN (1999). Global Public Goods: International Cooperation in the XXI Century. Publicado por Oxford University Press para el PNUD.

KAUL, PAUL, CONCEICAO, K. LE GOULVEN and R.U. MENDOZA (Eds.) (2003). Providing Global Public Goods: Managing globalization. New York: Oxford University Press, 131

KEOHANE, ROBERT (1984). After Hegemony: Cooperation and Discord in the World Political Economy. Princeton, NJ: Princeton University Press.

KIRTON, JOHN (2013): G20 Governance for a Globalized World. Asgate.

KRASNER, STEPHEN (editor) (1983). International Regimes. Cornell University Press. 
KEOHANE, ROBERT (2003). "Global Governance and Accountability" en David Held y Mathias Koenig-Archibugi (coordinadores): Taming Globalization: Frontiers of Governance. Oxford: Polity Press, pp. 130-159.

LAMY, PASCAL (2008). "The WTO's contribution to Global Governance" en Sampson (editor) The WTO and Global Governance. United Nations University Press, New York, pp: 39-57.

MORENO, PABLO (2013). The Metamorphosis of the IMF (2009-2011). Estudios Económicos, No. 78. Banco de España.

OCAMPO, JOSE ANTONIO (2010). "Rethinking Global Economic and Social Governance", Journal of Globalization and Development: Vol. 1: Issue. 1, Article 6.

RODRIK, DANI (2000). "How far will international Economic Integration go?" Journal of Economic Perspectives, Vol. 14, No. 1; invierno, pp. 177-186.

RODRIK, DANI (2011). The Globalization Paradox Democracy and the Future of the World Economy. W.W. Norton.

ROUBINI, NOURIEL y STEPHEN MIHM (2010). Crisis Economics. Allen Lane.

SAMUELSON, PAUL (1954). "The Pure Theory of Public Expenditure" Review of Economics and Statistics 36 (noviembre), pp. 387-89.

STEINBERG, FEDERICO (2011). "Para qué sirve el G-20 y qué ha conseguido" en Claves de la economía mundial 2011. ICEX-ICEI, Madrid.

STEINBERG, FEDERICO (2013). "La guerra de divisas como síntoma de los problemas del Sistema Monetario Internacional" Cuadernos de información económica 235. Julio/Agosto.

STIGLITZ, JOSEPH (2006). Making Globalization Work. W.W. Norton, New York.

STIGLITZ, JOSEPH y MARY KALDOR (editores) (2013). Quest for Security. Protection without Protectionsim and the Challenge of Global Governance. Columbia University Press.

TRUMAN, E.M. (2014). "IMF Reform Is Waiting on the United States". Policy Brief in International Economics 14-9. Peterson Institute for International Economics.

WOLF, MARTIN (2009): Fixing Global Finance. New Haven, Yale University Press.

WOODS, NGAIRE (2013). "The G-20 and Global Governance", en Stiglitz y Kaldor (2013).

ZAKARIA, FAREED (2008). The Post American World. W.W Norton \& Company Inc., Nueva York. 
